\title{
Promise and Pitfalls of Animal Models of Schizophrenia
}

\author{
David Feifel • Paul D. Shilling
}

Published online: 11 June 2010

(C) The Author(s) 2010. This article is published with open access at Springerlink.com

\begin{abstract}
Animal models are indispensible tools for advancing understanding of the cause of any given disease and developing new treatments. Developing animal models for schizophrenia presents formidable challenges owing to the distinctively human nature of the symptoms that define it and the thus-far-obscured underlying biological mechanisms. Nevertheless, progress has been and continues to be made in this important field of endeavor. This article discusses the challenges facing investigators who seek to develop and use animal models for translational research in schizophrenia and the responses that have emerged to those challenges, as well as the likely pathways that will lead to future progress.
\end{abstract}

Keywords Animal models · Schizophrenia · Psychosis · Antipsychotics

\section{Introduction}

Animal models are indispensable tools in the service of modern medicine. The conceptual paradigm of disease in modern medicine is based on a causal assumption that can be illustrated as follows:

Etiology $\rightarrow$ Biological mechanism $\rightarrow$ Signs/Symptoms

An etiology is an inducing condition that may be transitory in nature and temporally remote from the clinical manifestation of the disease (eg, prenatal exposure),

D. Feifel $(\bowtie) \cdot$ P. D. Shilling

Department of Psychiatry, University of California,

San Diego, 200 West Arbor Drive,

San Diego, CA 92103-8218, USA

e-mail: dfeifel@ucsd.edu whereas the mechanism refers to the consequent underlying biological perturbations that are contemporaneous with the signs and symptoms. Although it is represented in the schematic above as a unitary process, biological mechanism may be more accurately considered to be a manifold process that includes several subcomponents. Similarly, the defining signs and symptoms of disease are often manifold (eg, syndromatic).

The principle underlying the use of animal models to expand medical knowledge is that an animal model is a tool investigators can use to leverage what is known about a disease to discover unknown aspects of the disease. Therefore, the anchor that connects the animal to the human disease is always what is already known about the latter. This disease-oriented use of animal models is distinct from the "toxicologic" use of animal models in medical research, in which a putative toxin is administered, and pathological changes are screened broadly.

Based on the disease-oriented approach, animal models can aid discovery in medicine in three broad ways: 1) discovering the etiology of a specific disease, 2) discovering the biological mechanism underlying a specific disease, and 3) identifying and/or evaluating new treatments (eg, drugs) or treatment targets (eg, receptors).

As discussed in the subsequent sections of this article, investigators intending to exploit this paradigm in the service of advancing knowledge about schizophrenia face a formidable, although not insurmountable challenge because little other than the symptoms of this disease has been firmly established, and there are major barriers to modeling those symptoms. As a result, investigators have turned to alternate approaches, including substituting endophenotypes for symptoms and putative mechanisms for established mechanisms. 


\section{Modeling the Symptoms of Schizophrenia}

When an animal exhibits a phenotypic feature that resembles a phenotypic feature of a medical disease, the model is said to have face validity for that disease, at least with respect to that specific phenotype. The stronger the fidelity that exists between the human and animal phenotype, the stronger the face validity. As psychiatric diseases such as schizophrenia are essentially defined by their characteristic symptoms, most notably those delineated in the DSM-IV [1], intuitively, the most valuable type of face validity an animal model could exhibit would be face validity for these disease-defining symptoms. As discussed subsequently, face validity can be established for phenotypes other than the characteristic symptoms, but this strategy poses risks.

The $D S M-I V$ describes five core features of schizophrenia [1]. The first four-delusions, hallucinations, disorganized speech, and disorganized or catatonic behavior - are categorized as "positive" symptoms. The final feature is negative symptoms, which the DSM-IV indicates include affective flattening, alogia (poverty of speech), and avolition (poverty of volitional behavior). It becomes immediately apparent from the list of core features that developing animal models with good face validity for the defining symptoms of schizophrenia is highly problematic. Hallucinations and delusions are subjective phenomena, and without language or a reliable and readily measurable neurobiological marker for these internal phenomena, it is not possible to identify with certitude when an animal may be experiencing these states or even something analogous. It goes without saying that the symptom of disorganized speech cannot be modeled in infrahumans, owing to the unique status of human language and our limited ability to decipher vocal communications among animals. The fourth positive symptom described in the DSM-IV, disorganized or catatonic behavior, can in principle be modeled in animals; however, on its own, this is feature is not highly schizophrenia specific. One can easily envision an animal such as a rodent exhibiting, for example, grossly disorganized behavior as a result of a host of neural or neuromuscular perturbations that have little relevance to the higher order thought process disturbance that characterizes schizophrenia.

With regard to negative symptoms, only avolition is potentially modelable in animals (eg, by animals who display decreased motivation for normal social interactions). Indeed, social behavioral deficits induced by the administration of phencyclidine, an $N$-methyl-D-aspartate receptor antagonist, have been proposed as face valid models of negative symptoms [2]. However, avolition and the other negative symptoms are also seen in depression and therefore are not specific to schizophrenia. Thus, it would be difficult to assign strong validity for schizophrenia to an animal model that only exhibited negative features without some other coexpressed features that might link it more specifically to schizophrenia. On the whole, the ability to model the characteristic and defining features of schizophrenia is severely hampered because these features are not modelable in animals in any practical manner currently known, or they are potentially modelable but are, by themselves, not specific to schizophrenia.

It should be noted that some controversy exists regarding the importance of face validity, particularly face validity for the defining symptoms of schizophrenia, with some arguing that it is of primary importance [3], and others that it is the least important type of validity and perhaps not important at all [4]. We tend to the former opinion. As stated previously, the starting point in developing useful animal models for a medical disease is what is firmly known about the disease. Face validity for a disease may decline in importance when there exists a fairly firm understanding of the underlying etiology or mechanism. In that case, the characteristic symptoms of the disease can be confidently replaced by the well-established mechanistic processes as the anchor for development of animal models. For example, if elevated mesolimbic dopamine transmission was firmly established to be the underlying cause of positive symptoms in schizophrenia patients, then rats with elevated mesolimbic dopamine would be a strongly valid model of the disease, even if this produced behaviors in the rats (eg, excessive locomotor activity) that are not homologous to positive symptoms in schizophrenia patients.

However, until such a level of understanding regarding the underlying causes of schizophrenia is achieved, the clinical features of schizophrenia are arguably the only things that are currently firmly known about the disease (vide infra). For all intents and purposes then, they are equivalent to its definition. As such, the inherent difficulties establishing face validity are a very significant challenge to the enterprise of developing useful animal models of this disease, but as seen subsequently, alternate strategies have been adopted, namely modeling nonsymptom phenotypes, or endophenotypes. These strategies offer greater potential opportunities to establish animal models with face validity, albeit for these nonsymptom phenotypes.

\section{Modeling Underlying Causes of Schizophrenia}

An animal model that replicates the established etiology or mechanism underlying a disease is commonly described as having etiologic validity [4, 5••]. Although the term mechanistic validity is not in use, we believe such a term should be adopted, as it would be useful to distinguish an animal that modeled the inducing conditions of the disease (etiologic validity), which may be temporally remote and 
transitory relative to the clinical manifestations (eg, in utero toxin exposure), from an animal that modeled the mechanism that is contemporaneous with the clinical features and is believed to sustain their expression. Thus, the 1-methyl-4-phenyl-1,2,3,6-tetrahydropyridine (MPTP) animal model of Parkinson's disease could be considered to have mechanistic validity for Parkinson's disease due to the damage to the substantia nigra in Parkinson's disease sufferers and MPTP-treated animals. However, this model clearly does not have etiologic validity because MPTP is not the cause of idiopathic Parkinson's disease [6].

Notwithstanding an abundance of theories and putative candidates, neither the etiology nor the biological mechanism of schizophrenia is known with any certitude, except that the mechanism ultimately involves a disturbance in central nervous system function $[7 \bullet, 8 \bullet \bullet$. All mechanisms are speculative at present. Even the most well-developed mechanistic construct, the dopamine hypothesis, is just that - a hypothesis - as pathophysiologic evidence for increased dopamine transmission in schizophrenia remains sparse and inconclusive [9]. The strongest support for this theory is circumstantial, the ability of dopamine-2 (D2)receptor antagonists to ameliorate some of the symptoms [10] and the tendency of dopamine agonists such as amphetamine to induce symptoms similar to positive psychotic symptoms in nonschizophrenics. However, drugs may produce amelioration or induction of disease symptoms by altering biological systems that are not themselves directly involved in the development of the disease but can nevertheless modulate those systems. As a result of the current state of understanding, it can be concluded that etiologic (and mechanistic) validity for schizophrenia in an animal model is not presently an achievable goal.

\section{Employing Alternate Strategies}

Because of the challenges to modeling in animals the underlying causes and characteristic symptoms of schizophrenia, researchers have sought out alternative strategies.

These strategies include modeling nonsymptom phenotypes of schizophrenia that may be inherently more modelable in animals and modeling putative mechanisms and etiologies in lieu of established ones.

Modeling Endophenotypes as an Alternative to Symptoms

An endophenotype literally means an "inside" phenotype and refers to phenotypes that are not visible by the conventional examination used to observe or elicit the characteristic signs or symptoms of a disease. Thus, unlike grossly disorganized speech or bizarre delusions, which are readily observed or elicited through a routine clinical interview, endophenotypes are accessible only by specific and specialized testing. Several desirable characteristics have been established to help identify optimal schizophrenia endophenotypes for study and modeling from among the larger set of putative biomarkers for this disease $[11 \bullet, 12]$. These include strong heritability and presence in nonaffected relatives of schizophrenia patients at a higher rate than in the general population. The two categories of schizophrenia endophenotypes that have received the most attention with respect to modeling in animals are neurophysiologic endophenotypes and cognitive endophenotypes.

Several neurophysiologically based endophenotypes for schizophrenia have been identified and shown to be highly replicable, including reduced prepulse inhibition of the startle reflex (PPI), reduced P50 auditory evoked potential suppression, and abnormal smooth eye pursuit. A comprehensive discussion of these endophenotypes is beyond the scope of this article but can be found elsewhere [13]. PPI and P50 suppression are considered operational measures of information "gating," a normal function of the central nervous system whereby information is selectively filtered. Information gating is believed to be perturbed in individuals with schizophrenia, and some experts have proposed that this is of fundamental causal importance to the symptomatic expression of the disease [14]. The major advantage of these gating endophenotypes is that they can be successfully modeled in animals, often with strong homology to the human version and under similar parametric conditions [15]. Thus, establishing strong face validity for these endophenotypes is much more achievable than it is for the disease-defining symptoms, and most claims that an animal model has face validity for schizophrenia are in fact more precisely referring to face validity for one or more endophenotypes of the disease.

Another set of well-documented endophenotypes associated with schizophrenia are deficits in certain domains of cognitive functioning. Seven specific domains of cognitive functioning have been identified as consistently deficient in schizophrenia patients by the Measurement and Treatment Research to Improve Cognition in Schizophrenia (MATRICS) committee, a National Institute of Mental Health-sponsored initiative [16, 17]. These domains are working memory, verbal memory, visual learning, attention/vigilance, abstract reasoning, social cognition, and speed of processing. MATRICS has also identified specific neuropsychological tests that are best suited to assess each of these domains of cognitive dysfunction [18].

There is a long history of assessing cognitive performance in infrahuman species, including areas that are analogous to most of the domains of human cognition that are deficient in schizophrenia [19, 20]. Thus, modeling cognitive deficits associated with schizophrenia in animals is generally believed to be a very useful strategy, and 
substantial effort is being expended to refine existing animal tests and develop novel ones that have validity for domains of cognitive performance identified by MATRICS $[21,22]$. Cognitive endophenotypes generally cannot be modeled with the same degree of cross-species homology that is possible for neurophysiologic endophenotypes such as PPI and P50 suppression, in large part because many of the human tests of cognition rely on verbal communication. However, cognitive endophenotypes have an advantage in that they are justifiably considered more than a biomarker or surrogate phenotype for the defining symptoms of schizophrenia; rather, they are generally considered to be an unofficial (ie, not part of the DSM-IV criteria) third clinical syndrome of the disease (together with the positive and negative symptoms) and an important target for clinical remediation in their own right. As such, cognitive deficits often are not described as endophenotypes of schizophrenia as much as clinical features of the disease. Indeed, cognitive deficits have been shown to contribute significantly to morbidity and disability independent of positive and negative symptoms [23, 24]. Nevertheless, we have included them as endophenotypes of schizophrenia in this article because they are not part of the official $D S M$ clinical definition of the disease and thus are not factored into the diagnosis. Furthermore, they cannot be assessed with the normal clinical examination used routinely to determine the diagnosis of schizophrenia (eg, observation, interview, history). Instead, they require specialized and specific tests.

Although modeling endophenotypes of schizophrenia in lieu of characteristic symptoms provides a promising and viable alternative strategy to the bleak prospects of modeling symptoms of the disease, the endophenotype strategy is laden with pitfalls as well. In general, the endophenotypes identified in schizophrenia and used to develop animal models lack specificity for schizophrenia. For example, PPI has been shown to be reduced in patients with many neuropsychiatric and some non-neuropsychiatric diseases [25•]. Similarly, the cognitive deficits described among schizophrenia patients are also present in many brain-based diseases. This then raises the question of exactly what disease is an animal with gating or cognitive endophenotypes modeling?

Another related problem with relying on animal models that have face validity for an endophenotype of schizophrenia derives from our lack of understanding of the causal relationship that most endophenotypes of schizophrenia have to the pathological processes and symptoms of the disease. An endophenotype may be associated with schizophrenia in several ways, and it is possible that any given endophenotype may have an epiphenomenal relationship to the causal pathway linking etiology, mechanism, and symptoms of schizophrenia. If an endophenotype is an epiphenomenon and not directly and intrinsically connected through a causal relationship to the disease mechanism, then information gleaned from the study of that endophenotype in animals may produce misleading knowledge. For example, it is assumed that deficient PPI, as an indicator of dysfunctional sensorimotor gating, is intrinsically connected to the pathophysiologic changes in schizophrenia that give rise to the clinical features of the disease. However, it has been difficult to link abnormal PPI consistently with any clinical manifestation of schizophrenia, such as positive or negative symptoms or even cognitive deficits [25•]. Sometimes even endophenotypes with apparently compelling causal connections to the clinical manifestation can present potential pitfalls for investigators if the causal links are not firmly established. For example, amyloid-containing brain plaques are the most salient pathophysiologic finding in Alzheimer's disease. The plaques represent an obvious endophenotype to model in animals, and this strategy has been widely used by Alzheimer investigators. However, autopsy studies have revealed that some people with significant amyloid plaque build-up do not develop Alzheimer's disease. Thus far, studies have not consistently shown that reducing these plaques leads to cognitive improvement in patients with Alzheimer's disease [26].

\section{Modeling Etiologic and Mechanistic Constructs}

As previously stated, neither the etiology nor biological mechanism underlying schizophrenia is known; thus, establishing strong etiologic or mechanistic validity is not currently possible. Animal models that incorporate a mechanistic or etiological construct of schizophrenia are deemed to have construct validity, a term often used interchangeably with etiologic validity but what in fact is a distinct type of validity. The term construct validity connotes a theoretical aspect and reflects the speculative nature of the mechanisms modeled. In some cases, a single modeled feature can represent both face and construct validity, but it is important to recognize that the two types of validity are not necessarily linked. For example, a rat strain that has naturally reduced PPI exhibits construct validity regarding the sensorimotor gating deficits that are theorized to be linked to the neural disturbance and the clinical manifestation of schizophrenia (the disrupted sensorimotor construct of schizophrenia). It also exhibits face validity for PPI disruption, as the PPI abnormality in these rats is homologous to the PPI abnormality in schizophrenia patients and is elicited and measured using identical parametric conditions. On the other hand, amphetamine-induced hyperlocomotion, the basis of many animal models of schizophrenia, may exhibit construct validity for the excessive dopamine theory of schizophre- 
nia, but not face validity, because increased locomotor activity is not an established characteristic feature of schizophrenia [27].

\section{Enhancing Disease Relevance: Multiple Traits, Predictive Validity, and Temporal Validity}

The potential pitfalls posed by the need to model endophenotypes and etiologic/mechanistic constructs of schizophrenia can be mitigated in certain ways. One way is to develop animal models with multiple endophenotypes and mechanistic constructs associated with schizophrenia. Although any single schizophrenia endophenotype may lack specificity for schizophrenia, the coexistence of several such endophenotypes in a single model increases the likely relevance of the model for schizophrenia. Therefore, an animal model that exhibits decreased PPI and P50 suppression and poor performance on tests of vigilance and working memory will engender more confidence in its validity for schizophrenia than an animal model that only exhibits one of these features.

The extent to which an animal model responds to manipulations in a way that is consistent with the human disease it is modeling determines the extent to which it exhibits predictive validity. For animal models of schizophrenia, predictive validity most commonly refers to the animal model's ability to respond selectively to antipsychotic drugs $[4,5 \cdot 0]$. The establishment of predictive validity for an animal model is very important for the purpose of establishing it as a useful tool to discover new treatments $[4,5 \cdot \bullet]$. However, like multiple-modeled traits, establishing strong predictive validity can also generally mitigate the inherent weaknesses of having to rely on modeled endophenotypes and/or mechanistic constructs whose causal connection to the core mechanisms and symptoms of schizophrenia are speculative. Thus, in an animal model exhibiting PPI deficits, demonstration that the PPI deficits are selectively ameliorated by antipsychotics, but not non-antipsychotic psychotropics, enhances the overall validity of the model. All currently established antipsychotics share the ability to antagonize transmission through D2-receptors. Therefore, there is a risk that an animal model with apparent predictive validity may be functioning as nothing more than an in vivo assay for D2receptor affinity. For this reason, it is important to develop animal models that reflect known aspects of antipsychotic efficacy that transcend simple dopamine receptor affinity. Clozapine has relatively weak D2 binding affinity among established antipsychotics but is recognized as the most efficacious antipsychotic [28]. Furthermore, antipsychotic efficacy in schizophrenia patients is understood to begin with the first dose ingested and to grow in strength with repeated doses until it reaches a plateau after several weeks of daily use [29]. Thus, predictive validity in an animal model of schizophrenia can be strengthened beyond demonstrating simple responsiveness to acute antipsychotic administration if it 1) exhibits a response that is at least as strong (and preferably stronger) to clozapine as it is to more potent and selective D2 antagonists such as haloperidol and 2) exhibits a stronger response to repeated administration over time than it does to acute administration.

Evidence strongly implicates a genetic contribution to schizophrenia and subtle prodromal abnormalities before the full emergence of symptoms, which occurs in most cases 5 to 10 years after puberty. This distinct temporal feature of latency between inducing conditions and full manifestation of the disease affords another opportunity to strengthen the validity of an animal modeling for schizophrenia if this feature can be modeled [30].

A comprehensive review of animal models with potential relevance to schizophrenia is beyond the scope of this article, but in Table 1, we present a comparison of four rodent models that represent different inducing strategies: environmental, pharmacologic, neuroanatomic, and genetic.

\section{Future Directions}

Despite the prodigious challenges facing investigators in their efforts to develop better schizophrenia-relevant animal models, there is good reason to be optimistic that progress will continue to be made in developing animal models with stronger validity and utility. Sources of such advancements are most likely to derive from findings emanating from the study of schizophrenia patients themselves, which will lead to advances in developing animal models through the process of back translation. For example, identification of additional endophenotypes or certain aspects of currently known endophenotypes that have greater specificity for schizophrenia will provide new targets to model in animals that have stronger relevance to schizophrenia. Identification of efficacious drugs with novel mechanisms that do not rely on D2-receptor antagonism could also provide new tools to evaluate the predictive validity of animal models and thereby differentiate those that have specific predictive validity for schizophrenia from those whose apparent validity was spurious.

\section{Conclusions}

With no definitive knowledge of the mechanisms underlying schizophrenia and no realistic prospect of modeling its characteristic symptoms, the effort to model schizophrenia in infrahumans might appear futile. However, alternate 


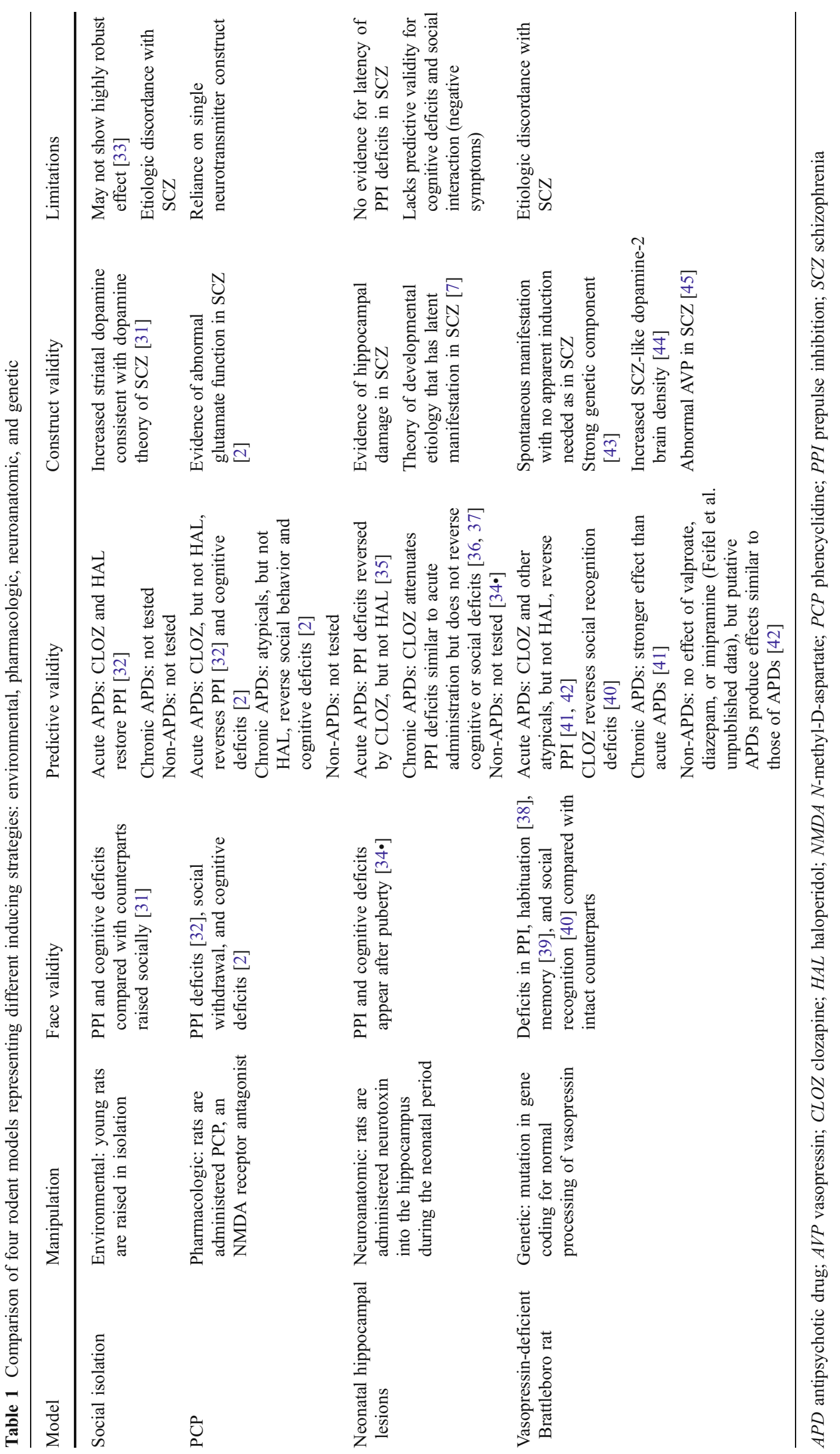


strategies are available that salvage this endeavor. These strategies include modeling endophenotypes of schizophrenia as a surrogate for disease-defining symptoms and modeling putative mechanisms and etiologies (mechanistic and etiologic constructs) in lieu of established ones. To the extent that these alternate strategies rely on unsubstantiated causal assumptions about these endophenotypes and constructs, they introduce risks of attributing validity to animal models with low relevance to essential features of schizophrenia. However, these risks can be mitigated by developing animal models that incorporate multiple endophenotypic and/or construct similarities rather than just a single one, thereby increasing the likelihood of their relevance to schizophrenia. In addition, demonstration of predictive validity can buttress confidence in the validity of animal models that rely on mechanistic or etiologic constructs and endophenotypes. This is particularly relevant if the predictive validity incorporates established nuanced features of antipsychotic drug efficacy, such as the differential efficacies and the therapeutic time course seen in schizophrenia patients. Back translation of future findings about schizophrenia from patients who suffer from it will provide opportunities to develop animal models with stronger validity and usefulness.

Acknowledgments Drs. Feifel and Shilling were partially supported by National Institute of Mental Health grants MH070700 and MH080910. The authors would like to thank Gilia Melendez and Michael O'Connor for proofreading this manuscript.

Disclosure No potential conflicts of interest relevant to this article were reported.

Open Access This article is distributed under the terms of the Creative Commons Attribution Noncommercial License which permits any noncommercial use, distribution, and reproduction in any medium, provided the original author(s) and source are credited.

\section{References}

Papers of particular interest, published recently, have been highlighted as:

- Of importance

•. Of major importance

1. American Psychiatric Association: Diagnostic and Statistical Manual of Mental Disorders, edn 4 (text revision). Washington, DC: American Psychiatric Association; 2000.

2. Mouri A, Noda Y, Enomoto T, Nabeshima T: Phencyclidine animal models of schizophrenia: approaches from abnormality of glutamatergic neurotransmission and neurodevelopment. Neurochem Int 2007, 51:173-184.
3. Holmes PV: Rodent models of depression: reexamining validity without anthropomorphic inference. Crit Rev Neurobiol 2003, 15:143-174.

4. Geyer MA, Markou A: The role of preclinical models in the development of psychotropic drugs. In Neuropsychopharmacology: The Fifth Generation of Progress. Edited by Davis KL, Charney D, Coyle JT, Nemeroff CB. Washington, DC: American College of Neuropsychopharmacology; 2002:445-455

5. - van der Staay FJ, Arndt SS, Nordquist RE: Evaluation of animal models of neurobehavioral disorders. Behav Brain Funct 2009, 5:11. This article addresses systematic approaches to the development and evaluation of animal models used to investigate neuropsychiatric disorders.

6. da Conceicao FS, Ngo-Abdalla S, Houzel JC, Rehen SK: Murine model for Parkinson's disease: from 6-OH dopamine lesion to behavioral test. J Vis Exp 2010, 35:1376.

7. - Keshavan MS, Tandon R, Boutros NN, Nasrallah HA: Schizophrenia, "just the facts": what we know in 2008. Part 3: neurobiology. Schizophr Res 2008, 106:89-107. This article describes potential intermediate phenotypes that may reflect "diverse pathological pathways from the phenome to genome" that could facilitate the discovery of novel etiologies and drug targets for schizophrenia.

8. •- Tandon R, Keshavan MS, Nasrallah HA: Schizophrenia, "just the facts": what we know in 2008. Part 1: overview. Schizophr Res 2008, 100:4-19. A total of 77 major findings in schizophrenia research are described and organized in terms of their relevance to etiology, pathophysiology, symptomatology, and treatment.

9. Tamminga CA, Holcomb HH: Phenotype of schizophrenia: a review and formulation. Mol Psychiatry 2005, 10:27-39.

10. Seeman MV: Pharmacologic features and effects of neuroleptics. Can Med Assoc J 1981, 125:821-826.

11. • Braff DL, Freedman R, Schork NJ, Gottesman II: Deconstructing schizophrenia: an overview of the use of endophenotypes in order to understand a complex disorder. Schizophr Bull 2007, 33:21-32. This article summarizes progress in the use of endophenotypes in the identification of the genetics and potential drug targets for schizophrenia.

12. Gottesman, II, Gould TD: The endophenotype concept in psychiatry: etymology and strategic intentions. Am J Psychiatry 2003, 160:636-645.

13. Turetsky BI, Calkins ME, Light GA, et al.: Neurophysiological endophenotypes of schizophrenia: the viability of selected candidate measures. Schizophr Bull 2007, 33:69-94.

14. Braff DL, Geyer MA: Sensorimotor gating and schizophrenia. Human and animal model studies. Arch Gen Psychiatry 1990, 47:181-188.

15. Swerdlow NR, Braff DL, Taaid N, Geyer MA: Assessing the validity of an animal model of deficient sensorimotor gating in schizophrenic patients. Arch Gen Psychiatry 1994, 51:139154.

16. Marder SR, Fenton W: Measurement and Treatment Research to Improve Cognition in Schizophrenia: NIMH MATRICS initiative to support the development of agents for improving cognition in schizophrenia. Schizophr Res 2004, 72:5-9.

17. Nuechterlein KH, Barch DM, Gold JM, et al.: Identification of separable cognitive factors in schizophrenia. Schizophr Res 2004, 72:29-39.

18. Nuechterlein KH, Green MF, Kern RS, et al.: The MATRICS Consensus Cognitive Battery, part 1: test selection, reliability, and validity. Am J Psychiatry 2008, 165:203-213.

19. Geyer MA, Moghaddam B: Animal models relevant to schizophrenia disorders. In Neuropsychopharmacology: The Fifth Generation of Progress. Edited by Davis KL, Charney D, Coyle JT, Nemeroff C. Washington, DC: American College of Neuropsychopharmacology; 2002:689-701. 
20. Tordjman S, Drapier D, Bonnot O, et al.: Animal models relevant to schizophrenia and autism: validity and limitations. Behav Genet 2007, 37:61-78.

21. Nuechterlein KH, Robbins TW, Einat H: Distinguishing separable domains of cognition in human and animal studies: what separations are optimal for targeting interventions? A summary of recommendations from breakout group 2 at the measurement and treatment research to improve cognition in schizophrenia new approaches conference. Schizophr Bull 2005, 31:870-874.

22. Young JW, Powell SB, Risbrough V, et al.: Using the MATRICS to guide development of a preclinical cognitive test battery for research in schizophrenia. Pharmacol Ther 2009, 122:150-202.

23. Tamminga CA, Buchanan RW, Gold JM: The role of negative symptoms and cognitive dysfunction in schizophrenia outcome. Int Clin Psychopharmacol 1998, 13(Suppl 3):S21-S26.

24. Meltzer HY: Cognitive factors in schizophrenia: causes, impact, and treatment. CNS Spectr 2004, 9:15-24.

25. - Swerdlow NR, Weber M, Qu Y, et al.: Realistic expectations of prepulse inhibition in translational models for schizophrenia research. Psychopharmacology (Berl) 2008, 199:331-388. This article elucidates the strengths and weaknesses of PPI as a translational model for schizophrenia research.

26. Gandy S: The role of cerebral amyloid beta accumulation in common forms of Alzheimer disease. J Clin Invest 2005, 115:1121-1129.

27. Minassian A, Henry BL, Geyer MA, et al.: The quantitative assessment of motor activity in mania and schizophrenia. J Affect Disord 2010, 120:200-206.

28. Foussias G, Remington G: Antipsychotics and schizophrenia: from efficacy and effectiveness to clinical decision-making. Can J Psychiatry 2010, 55:117-125.

29. Lambert TJ: The medical care of people with psychosis. Med J Aust 2009, 190:171-172.

30. Van den Buuse M, Garner B, Koch M: Neurodevelopmental animal models of schizophrenia: effects on prepulse inhibition. Curr Mol Med 2003, 3:459-471.

31. Fone KC, Porkess MV: Behavioural and neurochemical effects of post-weaning social isolation in rodents-relevance to developmental neuropsychiatric disorders. Neurosci Biobehav Rev 2008, 32:1087-1102.

32. Geyer MA, Krebs-Thomson K, Braff DL, Swerdlow NR: Pharmacological studies of prepulse inhibition models of sensorimotor gating deficits in schizophrenia: a decade in review. Psychopharmacology (Berl) 2001, 156:117-154.

33. Weiss IC, Feldon J, Domeney AM: Isolation rearing-induced disruption of prepulse inhibition: further evidence for fragility of the response. Behav Pharmacol 1999, 10:139-149.
34. - Tseng KY, Chambers RA, Lipska BK: The neonatal ventral hippocampal lesion as a heuristic neurodevelopmental model of schizophrenia. Behav Brain Res 2009, 204:295-305. This article reviews the advantages and limitations of the neonatal ventral hippocampal model of schizophrenia, the most thoroughly characterized developmentally based model for this disorder.

35. Le Pen G, Moreau JL: Disruption of prepulse inhibition of startle reflex in a neurodevelopmental model of schizophrenia: reversal by clozapine, olanzapine and risperidone but not by haloperidol. Neuropsychopharmacology 2002, 27:1-11.

36. Rueter LE, Ballard ME, Gallagher KB, et al.: Chronic low dose risperidone and clozapine alleviate positive but not negative symptoms in the rat neonatal ventral hippocampal lesion model of schizophrenia. Psychopharmacology (Berl) 2004, 176:312-319.

37. Levin ED, Christopher NC: Effects of clozapine on memory function in the rat neonatal hippocampal lesion model of schizophrenia. Prog Neuropsychopharmacol Biol Psychiatry 2006, 30:223-229.

38. Feifel D, Priebe K: Vasopressin-deficient rats exhibit sensorimotor gating deficits that are reversed by subchronic haloperidol. Biol Psychiatry 2001, 50:425-433.

39. Laycock JF, Gartside IB, Chapman JT: A comparison of the learning abilities of Brattleboro rats with hereditary diabetes insipidus and Long-Evans rats using positively reinforced operant conditioning. Prog Brain Res 1983, 60:183-187.

40. Feifel D, Mexal S, Melendez G, et al.: The Brattleboro rat displays a natural deficit in social discrimination that is restored by clozapine and a neurotensin analog. Neuropsychopharmacology 2009, 34:2011-2018.

41. Feifel D, Melendez G, Priebe K, Shilling PD: The effects of chronic administration of established and putative antipsychotics on natural prepulse inhibition deficits in Brattleboro rats. Behav Brain Res 2007, 181:278-286.

42. Feifel D, Melendez G, Shilling PD: Reversal of sensorimotor gating deficits in Brattleboro rats by acute administration of clozapine and a neurotensin agonist, but not haloperidol: a potential predictive model for novel antipsychotic effects. Neuropsychopharmacology 2004, 29:731-738.

43. Feifel D, Priebe K: The effects of cross-fostering on inherent sensorimotor gating deficits exhibited by Brattleboro rats. J Gen Psychol 2007, 134:173-182.

44. Shilling PD, Kinkead B, Murray T, et al.: Upregulation of striatal dopamine-2 receptors in Brattleboro rats with prepulse inhibition deficits. Biol Psychiatry 2006, 60:1278-1281.

45. Linkowski P, Geenen V, Kerkhofs M, et al.: Cerebrospinal fluid neurophysins in affective illness and in schizophrenia. Eur Arch Psychiatry Neurol Sci 1984, 234:162-165. 